\title{
SUMS REPRESENTING FOURIER TRANSFORMS ${ }^{1}$
}

R. P. BOAS, JR.

1. Introduction. Duffin $[5,6]$ has given a method for obtaining a pair of Fourier transforms from series involving a given function $\phi(x)$. With a change of notation, his formal result is as follows. Let the Fourier sine transform be written with kernel $\sin 2 \pi x t$ :

$$
F(x)=\int_{0}^{\infty} \sin 2 \pi x t f(t) d t ;
$$

then

$$
\text { (1) } \sum_{n=0}^{\infty} \frac{(-1)^{n}}{2 x} \phi\left(\frac{2 n+1}{2 x}\right) \text { and } \sum_{n=0}^{\infty}(-1)^{n} \frac{2}{2 n+1} \phi\left(\frac{2 x}{2 n+1}\right)
$$

are sine transforms. Duffin has shown how this formal relationship may be validated under various restrictions on $\phi$ and various interpretations of the sums.

The purpose of this note is to point out that Duffin's reciprocal relation is formally equivalent to a special case of Poisson's summation formula from the theory of Fourier transforms. The formal calculation may be made rigorous by imposing sufficiently stringent restrictions; however, it is not easy to derive Duffin's sufficient conditions from known sufficient conditions for Poisson's formula [8, pp. $60 \mathrm{ff} . ; 1$, pp. 33 ff.; $2 ; 3 ; 4 ; 7$ ], and his direct approach seems preferable. On the other hand, one could derive criteria for Poisson's formula from Duffin's theorems.

By using a more general form of Poisson's formula we shall obtain a more general pair of transforms, and by specialization we obtain not only Duffin's formulas but additional ones of similar type, as well as formulas for cosine transforms. Only the formal calculations will be given here. By proceeding similarly with other summation formulas one could obtain pairs of transforms with other kernels.

2. A general formula. All our calculations will be purely formal. Let $\phi(x)$ be a given function,

$$
\Phi(x)=\int_{-\infty}^{\infty} e^{-2 \pi i u x} \phi(u) d u
$$

Received by the editors October 12, 1951.

${ }^{1}$ The author is a Fellow of the John Simon Guggenheim Memorial Foundation. 
its Fourier transform. Then the symmetric form of Poisson's formula is $^{2}$

$$
x^{-1 / 2} e^{\pi i a b} \sum_{n=-\infty}^{\infty} e^{2 n \pi a i} \phi\left(\frac{n+b}{x}\right)=x^{1 / 2} e^{-\pi i a b} \sum_{n=-\infty}^{\infty} e^{2 n \pi b i} \Phi[x(n-a)],
$$

where $x, a, b$ are arbitrary. If we replace $\Phi$ by the integral (2) we have

$$
\begin{aligned}
x^{-1} e^{2 \pi i a b} \sum_{n=-\infty}^{\infty} e^{2 n \pi a i} \phi & \left(\frac{n+b}{x}\right) \\
& =\sum_{n=-\infty}^{\infty} e^{2 n \pi b i} \int_{-\infty}^{\infty} e^{-2 \pi i u x(n-a)} \phi(u) d u \\
& =\sum_{n=-\infty}^{\infty} e^{2 n \pi b i} \frac{\operatorname{sgn}(n-a)}{n-a} \int_{-\infty}^{\infty} e^{-2 \pi i u x} \phi\left(\frac{u}{n-a}\right) d u
\end{aligned}
$$

with the understanding that $0 \leqq a<1$ and that if $a=0$ the term on the right with $n=0$ is $\int_{-\infty}^{\infty} \phi(u) d u$. If we now interchange integration and summation we have

$$
\begin{aligned}
x^{-1} e^{2 \pi i a b} \sum_{n=-\infty}^{\infty} e^{2 n \pi a i} \phi & \left(\frac{n+b}{x}\right) \\
& =\int_{-\infty}^{\infty} e^{-2 \pi i u x} \sum_{n=-\infty}^{\infty} e^{2 n \pi b i} \frac{\operatorname{sgn}(n-a)}{n-a} \phi\left(\frac{u}{n-a}\right) d u,
\end{aligned}
$$

$a \neq 0$, with the appropriate modification when $a=0$. In other words,

$$
e^{2 \pi i a b} \sum_{n=-\infty}^{\infty} e^{2 n \pi a i} \frac{1}{x} \phi\left(\frac{n+b}{x}\right)
$$

is the Fourier transform of

$$
\sum_{n=-\infty}^{\infty} e^{2 n \pi b i} \frac{\operatorname{sgn}(n-a)}{n-a} \phi\left(\frac{x}{n-a}\right)
$$

with the understanding that if $a=0$ the term with $n=0$ is omitted from (5) and (4) is diminished by $\int_{-\infty}^{\infty} \phi(u) d u$.

On the other hand, if we take the Fourier transform of (5) term

2 Formula (3) is easily obtained from the usual forms of Poisson's formula, but is not of ten given in this generality. I learned it from lectures by G. H. Hardy. It may be noted incidentally that the more bizarre variants of Poisson's formula such as those of Ramanujan quoted by Titchmarsh [8, p. 62] follow from (3) by specializing the parameters and require no separate proof. 
by term and retrace our steps we return to (3), so that (3) and (4), (5) are formally equivalent.

3. Special cases. (i) Let $\phi$ be odd, $a=b=1 / 2$. Then (4), (5) reduce to Duffin's pair (1) of sine transforms.

(ii) Let $\phi$ be odd and $a=0$. Then

$$
\sum_{n=-\infty}^{\infty} \frac{1}{2 x} \phi\left(\frac{n+b}{x}\right)
$$

is the sine transform of

$$
\sum_{n=1}^{\infty} \frac{2}{n} \phi\left(\frac{x}{n}\right) \sin 2 n \pi b ;
$$

and so, for example, if $b=1 / 3$,

$$
\frac{1}{2 x}\left\{\phi\left(\frac{1}{3 x}\right)-\phi\left(\frac{2}{3 x}\right)+\phi\left(\frac{4}{3 x}\right)-\phi\left(\frac{5}{3 x}\right)+\cdots\right\}
$$

is the sine transform of

$$
3^{1 / 2}\left\{\phi(x)-\frac{1}{2} \phi\left(\frac{x}{2}\right)+\frac{1}{4} \phi\left(\frac{x}{4}\right)-\frac{1}{5} \phi\left(\frac{x}{5}\right)+\cdots\right\} .
$$

(iii) Let $\phi$ be even and $a=b=0$. Then

$$
\frac{1}{x}\left\{\phi(0) / 2+\sum_{n=1}^{\infty} \phi\left(\frac{n}{x}\right)-\int_{0}^{\infty} \phi(u) d u\right\}
$$

is the cosine transform of

$$
\sum_{n=1}^{\infty} \frac{2}{n} \phi\left(\frac{x}{n}\right)
$$

(iv) Let $\phi$ be even, $a \neq 0, b=0$. Then

$$
\frac{1}{x}\left\{\phi(0) / 2+\sum_{n=1}^{\infty} \phi\left(\frac{n}{x}\right) \cos 2 \pi n a\right\}
$$

is the cosine transform of

$$
\sum_{n=1}^{\infty} \frac{1}{n-a} \phi\left(\frac{x}{n-a}\right)+\sum_{n=0}^{\infty} \frac{1}{n+a} \phi\left(\frac{x}{n+a}\right) ;
$$

and so, for example, if $a=1 / 2$,

$$
\frac{1}{x}\left\{\phi(0) / 2+\sum_{n=1}^{\infty}(-1)^{n} \phi\left(\frac{n}{x}\right)\right\}
$$


is the cosine transform of

$$
\sum_{n=0}^{\infty} \frac{4}{2 n+1} \phi\left(\frac{2 x}{2 n+1}\right)
$$

while if $a=1 / 3$,

$$
\begin{aligned}
\frac{1}{x}\left\{\phi(0)-\phi\left(\frac{1}{x}\right)-\phi\left(\frac{2}{x}\right)+2 \phi\left(\frac{3}{x}\right)\right. & -\phi\left(\frac{4}{x}\right) \\
& \left.-\phi\left(\frac{5}{x}\right)+2 \phi\left(\frac{6}{x}\right)+\cdots\right\}
\end{aligned}
$$

is the cosine transform of

$$
\begin{aligned}
6\left\{\phi(3 x)+\frac{1}{2} \phi\left(\frac{3 x}{2}\right)+\frac{1}{4} \phi\left(\frac{3 x}{4}\right)\right. & \\
& \left.+\frac{1}{5} \phi\left(\frac{3 x}{5}\right)+\frac{1}{7} \phi\left(\frac{3 x}{7}\right)+\cdots\right\} .
\end{aligned}
$$

\section{REFERENCES}

1. S. Bochner, Vorlesungen über Fouriersche Integrale, Leipzig, 1932.

2. R. P. Boas, Jr., Poisson's summation formula in $L^{2}$, J. London Math. Soc. vol. 21 (1946) pp. 102-105.

3. S. Borgen, Note on the summability of Poisson's formula, J. London Math. Soc. vol. 19 (1944) pp. 100-105.

4. - Note on Poisson's formula, J. London Math. Soc. vol. 19 (1944) pp. 213-219.

5. R. J. Duffin, Representation of Fourier integrals as sums. I, Bull. Amer. Math. Soc. vol. 51 (1945) pp. 447-455.

6. - Representation of Fourier integrals as sums. II, Proceedings of the American Mathematical Society vol. 1 (1950) pp. 250-255.

7. A. P. Guinand, On Poisson's summation formula, Ann. of Math. (2) vol. 42 (1941) pp. 591-603.

8. E. C. Titchmarsh, Introduction to the theory of Fourier integrals, Oxford, 1937.

NORTHWESTERN UNIVERSITY 